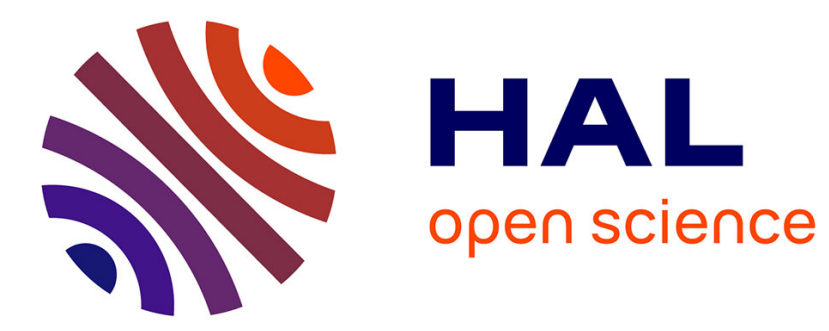

\title{
Conservative estimates of blocking and outage probabilities in CDMA networks
}

Thomas Bonald, Alexandre Proutière

\section{To cite this version:}

Thomas Bonald, Alexandre Proutière. Conservative estimates of blocking and outage probabilities in CDMA networks. Performance Evaluation, 2005, 10.1016/j.peva.2005.07.001 • hal-01244805

\section{HAL Id: hal-01244805 \\ https://hal.science/hal-01244805}

Submitted on 16 Dec 2015

HAL is a multi-disciplinary open access archive for the deposit and dissemination of scientific research documents, whether they are published or not. The documents may come from teaching and research institutions in France or abroad, or from public or private research centers.
L'archive ouverte pluridisciplinaire HAL, est destinée au dépôt et à la diffusion de documents scientifiques de niveau recherche, publiés ou non, émanant des établissements d'enseignement et de recherche français ou étrangers, des laboratoires publics ou privés. 


\title{
Conservative Estimates of Blocking and Outage Probabilities in CDMA Networks
}

\author{
T. Bonald, A. Proutière ${ }^{1}$ \\ France Telecom, Division R\&D, Issy-les-Moulineaux, France
}

\begin{abstract}
The outage probability is a key performance measure for real-time traffic in wireless networks, often considered as even more critical than the blocking probability. The blocking and outage probabilities do not have closed-form expressions as they depend in a too complex way on the traffic characteristics (call duration, bit rate requirement), the radio conditions (fading, shadowing, noise, interference) and the considered admission and outage policies. We derive upper bounds for the blocking and outage probabilities for general traffic characteristics and radio conditions under the mild assumption that the admission and outage policies satisfy a certain monotonicity property. These conservative estimates may depend on call characteristics like the bit rate requirement and the trajectory of the mobile. The results are applied to the uplink and the downlink of CDMA networks.
\end{abstract}

Key words: Blocking probability, outage probability, shot-noise process, CDMA.

\section{Introduction}

Third-generation cellular networks offer a wide variety of services including high-speed data transfers and audio/video streaming in addition to conventional voice services. While the packet delay tolerance of data transfers allows a flexible design of control mechanisms for data traffic, it is a real challenge to guarantee the quality of service of real-time applications throughout the call duration. A key role is played by admission control that blocks new calls when necessary to maintain the quality of ongoing calls. Such a control mechanism is not sufficient, however, and it is sometimes necessary to interrupt some calls due to the varying radio conditions and bit rate requirements of

1 The authors are also affiliated with Ecole Normale Supérieure, Paris.

Email: \{thomas.bonald,alexandre.proutiere\}@francetelecom.com

Preprint submitted to Elsevier Science 22 June 2005 
the mobiles. We say that such calls are in outage. The outage probability is a key performance measure, often considered as even more critical than the blocking probability. An "Erlang formula" predicting the blocking and outage probabilities would allow the development of optimal dimensioning rules and the design of efficient control mechanisms. The derivation of such a formula is out of reach, however, as it would depend in a too complex way on the traffic characteristics (call duration, possibly varying bit rate requirement) and the radio conditions (fading, shadowing, noise, interference).

The phenomenon of outage was first considered by Bock and Ebstein who applied linear programming techniques to derive the condition under which the signal-to-noise ratio (SNR) requirements of all users can be met [6]. The study of this so-called feasibility condition was later shown to be equivalent to an eigenvalue problem, solved using Perron-Frobenius theory [1], [14], [21]. Foschini-Miljanic power control algorithms were derived on this basis and proved to converge to the optimal solution for which the transmission powers are minimum [12]. These algorithms also have the useful property of diverging at an exponential rate when the feasibility condition is not satisfied, giving a quick indication of an outage situation where some calls must be interrupted. An intense research activity has since been devoted to the analysis of power control algorithms, see [5], [16] for instance. The resulting performance was first considered by Gilhousen et al. who studied the probability of an outage situation given statistics on the radio environment like fading and shadowing [13]. This so-called outage probability has since been evaluated for quite general models using standard statistical techniques like Chernoff bounds and Edgeworth expansions, see [8], [20] for instance.

It is worth noting that admission control is not taken into account in the above mentioned papers. Evans and Everitt proposed an admission control scheme based on the notion of effective bandwidth that guarantees a negligible outage probability [10], [11]. The effective bandwidth of a particular call depends on the radio conditions experienced during the entire call duration, however, which makes the admission control scheme hardly applicable in practice. The blocking probability resulting from more realistic schemes where the admission decision depends on the actual radio conditions was evaluated in [2], [4] using the reversibility of the underlying Markov process. But the radio conditions are then assumed to be static so that no outage occurs. In fact, work on the joint analysis of blocking and outage probabilities is extremely rare. A specific scheme where the admission of a call depends on the radio conditions in both the uplink and the downlink was considered in [15]. A more general model is considered in [3] but the result is derived under a specific independence assumption that is not justified in most cases.

It may be argued that the main difficulty arising in the study of a wireless network with time-varying radio conditions comes from the fact that the stochas- 
tic process describing the network state is not reversible. The blocking and outage probabilities are indeed completely different if, for the same marginal distribution, the radio conditions evolve in a different way (e.g. there is no outage in the ideal case where the radio conditions cannot worsen). Unlike reversible queueing networks whose stationary distribution is insensitive [7], the performance of the considered network depends on the exact traffic characteristics and radio conditions. Instead of evaluating network performance under specific assumptions, we derive upper bounds on the blocking and outage probabilities for general traffic characteristics and radio conditions. These conservative estimates may depend on call characteristics like the bit rate requirement, the call duration, the trajectory of the mobile, etc. In addition, we estimate the actual outage probability as perceived by users (the probability an arbitrary call is in outage) and not the non-feasibility probability (the probability at least one call is in outage). The results only rely on the assumption that the admission and outage policies satisfy a certain monotonicity property, as described below.

The model is described and the notions of monotonic admission and outage policies are introduced in the next section. We then derive conservative estimates of the blocking and outage probabilities. The results are applied to the uplink and the downlink of CDMA networks in Sections 4 and 5, respectively. Section 6 concludes the paper.

\section{Model}

\subsection{Traffic characteristics and radio conditions}

Consider a wireless network whose resources are shared by a random number of mobiles. Each mobile is characterized by its "state" which determines its location in the network, its radio conditions, its service requirement (e.g. voice or video), etc. We assume this state takes values in some finite set $\mathcal{L}=\{1, \ldots, L\}$. In particular, mobiles do not have continuous trajectories in the network but "jump" from one location to another. Note that an arbitrarily close approximation of any continuous trajectory may be obtained by increasing the number of states $L$.

Mobiles arrive in the network at random, attempt a call and leave the network immediately if blocked or persist until the end of the call if admitted. A mobile may suffer temporary periods of outage but do not abandon the call. This is a reasonable assumption when the outage probability is sufficiently low. Let $\mathcal{K}=\{1, \ldots, K\}$ be the set of mobile classes. Class- $k$ mobiles arrive in the network according to a Poisson process of intensity $\lambda^{k}>0$ and attempt 
a call of duration $\sigma^{k}>0$ characterized by some $\mathcal{L}$-valued, right-continuous, deterministic process of duration $\sigma^{k}$. Thus all class- $k$ mobiles have the same call characteristics (duration, trajectory in the network, successive bit rate requirements, etc.) Note that an arbitrarily close approximation of any distribution of call characteristics may be obtained by increasing the number of classes $K$.

Let $\rho^{k}=\lambda^{k} \sigma^{k}$ be the traffic intensity of class- $k$ mobiles (in erlangs). By Little's law, this corresponds to the mean number of class- $k$ mobiles in the network in the absence of any blocking. We denote by $\lambda$ the overall arrival rate and by $\rho$ the overall traffic intensity.

$$
\lambda=\sum_{k \in \mathcal{K}} \lambda^{k}, \quad \rho=\sum_{k \in \mathcal{K}} \rho^{k} .
$$

Let $\sigma_{l}^{k}$ be the mean time spent by a class- $k$ mobile in state $l$ during its call. We denote by $\rho_{l}^{k}=\lambda^{k} \sigma_{l}^{k}$ the corresponding traffic intensity. We have:

$$
\sigma^{k}=\sum_{l \in \mathcal{L}} \sigma_{l}^{k}, \quad \rho^{k}=\sum_{l \in \mathcal{L}} \rho_{l}^{k}
$$

Similarly, we denote by $\sigma_{l}$ the mean time spent by a mobile of arbitrary class in state $l$ in the absence of any blocking, and by $\rho_{l}=\lambda \sigma_{l}$ the corresponding traffic intensity. We have:

$$
\sigma_{l}=\frac{1}{\lambda} \sum_{k \in \mathcal{K}} \lambda_{k} \sigma_{l}^{k}, \quad \rho_{l}=\sum_{k \in \mathcal{K}} \rho_{l}^{k} .
$$

\subsection{Network resources}

Let $N(t)$ be the network state at time $t$ in the absence of any blocking. This is a $K L$-dimensional vector whose $k, l$-component $N_{l}^{k}(t)$ gives the number of class- $k$ mobiles in state $l$ at time $t$. We denote by $\mathcal{N}=\mathbb{N}^{K L}$ the corresponding state space. We use the following notation:

- For any $n \in \mathcal{N}$ and $k \in \mathcal{K}, n^{k}=\sum_{l \in \mathcal{L}} n_{l}^{k}$ is the number of class- $k$ mobiles.

- For any $n \in \mathcal{N}$ and $l \in \mathcal{L}, n_{l}=\sum_{k \in \mathcal{K}} n_{l}^{k}$ is the number of mobiles in state $l$.

- For any $n \in \mathcal{N},|n|=\sum_{k \in \mathcal{K}} n^{k}=\sum_{l \in \mathcal{L}} n_{l}$ is the total number of mobiles.

- For any $n, m \in \mathcal{N}, n \leq m$ means $n_{l}^{k} \leq m_{l}^{k}$ for all $k \in \mathcal{K}$ and $l \in \mathcal{L}$.

- For any $k \in \mathcal{K}$ and $l \in \mathcal{L}, e_{l}^{k}$ is the unit vector with 1 in component $k, l$ and 0 elsewhere.

Some network states $n \in \mathcal{N}$ are not admissible in the sense that the radio resources (frequency band, transmission power) are not sufficient to meet the service requirements of all mobiles given their location and radio conditions. We denote by $\mathcal{A} \subset \mathcal{N}$ the set of admissible network states. 
Let $\bar{N}(t)$ be the real network state at time $t$, whose $k, l$-component $\bar{N}_{l}^{k}(t)$ gives the number of admitted class- $k$ mobiles in state $l$ at time $t$. We assume the decision of admitting or blocking a new call attempted by a class- $k$ mobile in state $l$ at time $t$ depends on $k, l$ and $\bar{N}(t)$ only. Specifically, such a call is admitted if and only if $\bar{N}(t)$ belongs to some set $\mathcal{A}_{l}^{k} \subset \mathcal{N}$. One may choose $\mathcal{A}_{l}^{k}=\left\{n: n+e_{l}^{k} \in \mathcal{A}\right\}$, which means that a mobile is admitted if and only if the resulting network state is admissible. Such a greedy policy may simply be implemented by testing the convergence of the power control algorithms in the new network state. It may result in excessive outage, however, since the radio conditions may worsen or the bit rate requirements may increase. It may indeed be preferable to enforce stricter admission conditions, e.g. by keeping a margin on the transmission power of the mobiles (for the uplink) or the base stations (for the downlink). One may also imagine that the admission set $\mathcal{A}_{l}^{k}$ is in fact larger than the admissible set $\mathcal{A}$, which means that new calls may be admitted though some mobiles are in outage. This may be the case if some form of priority is given to a mobile class or a service type (e.g. voice over video). In fact, we do not make any assumption on the admission policy except for the following monotonicity property.

Assumption 1 (Monotonic admission policy) If a call is blocked in state $n \in \mathcal{N}$, it is also blocked in any state $m \in \mathcal{N}$ such that $m \geq n$.

As already mentioned, the real network state $\bar{N}(t)$ is not necessarily admissible at any time $t$ due to varying radio conditions and bit rate requirements. If the real network state is not admissible, the service of some mobiles must be temporarily interrupted to maintain the service of some other mobiles. We assume the vector of the numbers of mobiles of each class in each state in outage is a function $f(n)$ of the network state $n$ only. Note that $f(n)=0$ for all $n \in \mathcal{A}$, which means that no mobile is in outage if the network state is admissible, and $n-f(n) \in \mathcal{A}$ for all $n \in \mathcal{N}$, which means that mobiles that are not in outage receive service. One may wish to minimize the number of mobiles in outage (by interrupting those calls that consume more radio resources) or to favor some mobile classes (e.g. to interrupt video calls rather than voice calls). Again, we do not make any assumption on the outage policy except that it is monotonic in the sense that the function $f$ is itself monotonic, i.e. $f(n) \leq f(m)$ for all network states $n \leq m$.

Assumption 2 (Monotonic outage policy) If a mobile is in outage in state $n \in \mathcal{N}$, it is also in outage in any state $m \in \mathcal{N}$ such that $m \geq n$. 


\subsection{Performance metrics}

In the rest of the paper, we assume the network is in steady state so that we remove the explicit time dependency of the stochastic processes $\{N(t)\}_{t \geq 0}$ and $\{\bar{N}(t)\}_{t \geq 0}$. By the PASTA property, the blocking probability $\bar{B}^{k}$ of a class- $k$ mobile is given by:

$$
\bar{B}^{k}=\operatorname{Pr}\left[\bar{N} \notin \mathcal{A}_{l}^{k}\right]
$$

where $l$ denotes the initial state of class- $k$ mobiles. Let $\mathcal{K}_{l}$ be the set of classes $k$ whose initial state is $l$. We denote by $\lambda_{l}=\sum_{k \in \mathcal{K}_{l}} \lambda^{k}$ the arrival rate of mobiles in state $l$. The blocking probability $\bar{B}_{l}$ of a call starting in state $l$ and the overall blocking probability $\bar{B}$ are given by:

$$
\bar{B}_{l}=\frac{1}{\lambda_{l}} \sum_{k \in \mathcal{K}_{l}} \lambda^{k} \bar{B}^{k}, \quad \bar{B}=\frac{1}{\lambda} \sum_{k \in \mathcal{K}} \lambda^{k} \bar{B}^{k} .
$$

We refer to the outage probability as the probability an arbitrary admitted call is in outage. Since we assume the network is in steady state, this corresponds to the ratio of the mean time in outage to the mean call duration. Assume $\rho_{l}^{k}>0$ and $\bar{B}^{k}<1$ so that there are class- $k$ mobiles in state $l$ with positive probability. By Little's law, the outage probability $\bar{O}_{l}^{k}$ of a class- $k$ mobile in state $l$ is the ratio of the mean number of class- $k$ mobiles in state $l$ in outage to the mean number of class- $k$ mobiles in state $l$ :

$$
\bar{O}_{l}^{k}=\frac{\mathrm{E}\left[f(\bar{N})_{l}^{k}\right]}{\mathrm{E}\left[\bar{N}_{l}^{k}\right]}
$$

Using the fact that the actual arrival rate of class- $k$ mobiles is $\lambda^{k}\left(1-\bar{B}^{k}\right)$, it follows again by Little's law that the mean number of class- $k$ mobiles in state $l$ is:

$$
\mathrm{E}\left[\bar{N}_{l}^{k}\right]=\rho_{l}^{k}\left(1-\bar{B}^{k}\right)
$$

We deduce:

$$
\bar{O}_{l}^{k}=\frac{\mathrm{E}\left[f(\bar{N})_{l}^{k}\right]}{\rho_{l}^{k}\left(1-\bar{B}^{k}\right)} .
$$

Similarly, using the fact that

$\mathrm{E}\left[\bar{N}^{k}\right]=\rho^{k}\left(1-\bar{B}^{k}\right), \quad \mathrm{E}\left[\bar{N}_{l}\right]=\sum_{k \in \mathcal{K}} \rho_{l}^{k}\left(1-\bar{B}^{k}\right) \quad$ and $\quad \mathrm{E}[|\bar{N}|]=\sum_{k \in \mathcal{K}} \rho^{k}\left(1-\bar{B}^{k}\right)$,

we obtain the outage probability $\bar{O}^{k}$ of class- $k$ mobiles:

$$
\bar{O}^{k}=\frac{\mathrm{E}\left[f(\bar{N})^{k}\right]}{\rho^{k}\left(1-\bar{B}^{k}\right)}=\frac{1}{\rho^{k}} \sum_{l \in \mathcal{L}} \rho_{l}^{k} \bar{O}_{l}^{k},
$$


the outage probability $\bar{O}_{l}$ of a mobile in state $l$ :

$$
\bar{O}_{l}=\frac{\mathrm{E}\left[f(\bar{N})_{l}\right]}{\sum_{k \in \mathcal{K}} \rho_{l}^{k}\left(1-\bar{B}^{k}\right)}=\frac{\sum_{k \in \mathcal{K}} \rho_{l}^{k}\left(1-\bar{B}^{k}\right) \bar{O}_{l}^{k}}{\sum_{k \in \mathcal{K}} \rho_{l}^{k}\left(1-\bar{B}^{k}\right)}
$$

and the overall outage probability $\bar{O}$ :

$$
\bar{O}=\frac{\mathrm{E}[|f(\bar{N})|]}{\sum_{k \in \mathcal{K}} \rho^{k}\left(1-\bar{B}^{k}\right)}=\frac{\sum_{k \in \mathcal{K}} \rho^{k}\left(1-\bar{B}^{k}\right) \bar{O}^{k}}{\sum_{k \in \mathcal{K}} \rho^{k}\left(1-\bar{B}^{k}\right)} .
$$

\section{Conservative estimates}

The stationary distribution of the real network state $\bar{N}$ does not only depend on the marginal distributions of traffic characteristics and radio conditions but on their evolution, that is on the entire process describing the state of each call. This makes the evaluation of the blocking and outage probabilities intractable in general. We provide conservative estimates of these performance metrics through the study of the "free" process $\{N(t)\}_{t \geq 0}$ that describes the evolution of the network state in the absence of blocking.

\subsection{Free process}

The free process $\{N(t)\}_{t \geq 0}$ is a superposition of $K$ shot-noise processes, whose stationary distribution is given by:

$$
\pi(n)=e^{-\rho} \prod_{k \in \mathcal{K}} \prod_{l \in \mathcal{L}} \frac{\rho_{l}^{k^{n_{l}^{k}}}}{n_{l}^{k !}}
$$

It is worth noting that this stationary distribution depends on the traffic characteristics and the radio conditions through their marginal distributions only, determined by the traffic intensities $\rho_{l}^{k}, k \in \mathcal{K}, l \in \mathcal{L}$.

\subsection{Blocking probability}

We estimate the blocking probability of class- $k$ mobiles as:

$$
B^{k}=\operatorname{Pr}\left[N \notin \mathcal{A}_{l}^{k}\right]
$$

where $l$ denotes the initial state of class- $k$ mobiles. Similarly, the blocking probability of a mobile in state $l$ and the overall blocking probability are 
respectively estimated as:

$$
B_{l}=\frac{1}{\lambda_{l}} \sum_{k \in \mathcal{K}_{l}} \lambda^{k} B^{k}, \quad B=\frac{1}{\lambda} \sum_{k \in \mathcal{K}} \lambda^{k} B^{k}
$$

Proposition 1 We have for all mobile classes $k \in \mathcal{K}$ and all mobile states $l \in \mathcal{L}$ :

$$
\bar{B}^{k} \leq B^{k}, \quad \bar{B}_{l} \leq B_{l} \quad \text { and } \quad \bar{B} \leq B
$$

Proof. In view of (4) and (11), the first inequality follows from $\bar{N} \leq N$ and Assumption 1. The other two inequalities follow from (5) and (12).

It is worth noting that our conservative estimate of the blocking probability is closely related to the non-feasibility probability (cf. Section 1), defined as

$$
\operatorname{Pr}[N \notin \mathcal{A}]
$$

and often referred to as the outage probability. Specifically, the non-feasibility probability (13) corresponds to the blocking probability (11) of a call that does not require any radio resource (so that $\mathcal{A}_{l}^{k}=\mathcal{A}$ ).

\subsection{Outage probability}

We estimate the outage probability of a class- $k$ mobile in state $l$ as:

$$
O_{l}^{k}=\frac{\mathrm{E}\left[f(N)_{l}^{k}\right]}{\rho_{l}^{k}\left(1-B^{k}\right)}
$$

The outage probability of a class- $k$ mobile, the outage probability of a mobile in state $l$ and the overall outage probability are respectively estimated as:

$$
O^{k}=\frac{\mathrm{E}\left[f(N)^{k}\right]}{\rho^{k}\left(1-B^{k}\right)}, \quad O_{l}=\frac{\mathrm{E}\left[f(N)_{l}\right]}{\sum_{k \in \mathcal{K}} \rho_{l}^{k}\left(1-B^{k}\right)}, \quad O=\frac{\mathrm{E}[|f(N)|]}{\sum_{k \in \mathcal{K}} \rho^{k}\left(1-B^{k}\right)} .
$$

Proposition 2 We have for all mobile classes $k \in \mathcal{K}$ and all mobile states $l \in \mathcal{L}$ :

$$
\bar{O}_{l}^{k} \leq O_{l}^{k}, \quad \bar{O}^{k} \leq O^{k}, \quad \bar{O}_{l} \leq O_{l} \quad \text { and } \quad \bar{O} \leq O
$$

Proof. We deduce from the inequality $\bar{N} \leq N$ and Assumption 2 that $f(\bar{N})_{l}^{k} \leq$ $f(N)_{l}^{k}$. It then follows from Proposition 1 that:

$$
\bar{O}_{l}^{k}=\frac{\mathrm{E}\left[f(\bar{N})_{l}^{k}\right]}{\rho_{l}^{k}\left(1-\bar{B}_{l}^{k}\right)} \leq \frac{\mathrm{E}\left[f(N)_{l}^{k}\right]}{\rho_{l}^{k}\left(1-\bar{B}_{l}^{k}\right)} \leq \frac{\mathrm{E}\left[f(N)_{l}^{k}\right]}{\rho_{l}^{k}\left(1-B_{l}^{k}\right)}=O_{l}^{k}
$$


Similarly, we have $f(\bar{N})^{k} \leq f(N)^{k}, f(\bar{N})_{l} \leq f(N)_{l}$ and $|f(\bar{N})| \leq|f(N)|$ so that, in view of Proposition 1 ,

$$
\bar{O}^{k} \leq \frac{\mathrm{E}\left[f(N)^{k}\right]}{\rho^{k}\left(1-\bar{B}^{k}\right)} \leq O^{k}, \bar{O}_{l} \leq \frac{\mathrm{E}\left[f(N)_{l}\right]}{\sum_{k \in \mathcal{K}} \rho_{l}^{k}\left(1-\bar{B}^{k}\right)} \leq O_{l}, \bar{O} \leq \frac{\mathrm{E}[|f(N)|]}{\sum_{k \in \mathcal{K}} \rho^{k}\left(1-\bar{B}^{k}\right)} \leq O
$$

It follows from Propositions 1 and 2 that the considered estimates of the blocking and outage probabilities can be used to develop conservative dimensioning rules. The accuracy of the approximation (and the resulting margin in the derived dimensioning rules) depends on precise statistics of traffic characteristics (frequency of bit rate changes) and radio conditions (fading variations, mobility). The approximation is tight when the blocking probability tends to zero since the real network state $\bar{N}$ then coincides with the free process $N$. The estimates of the outage probabilities may then simply be written as:

$$
O_{l}^{k} \approx \frac{\mathrm{E}\left[f(N)_{l}^{k}\right]}{\rho_{l}^{k}}, \quad O^{k} \approx \frac{\mathrm{E}\left[f(N)^{k}\right]}{\rho^{k}}, \quad O_{l} \approx \frac{\mathrm{E}\left[f(N)_{l}\right]}{\rho_{l}} \quad \text { and } \quad O \approx \frac{\mathrm{E}[|f(N)|]}{\rho}
$$

We use these expressions in the rest of the paper.

\section{Application to the uplink}

We first apply the above results to the uplink of CDMA networks. We focus on a particular cell and assume for simplicity that inter-cell interference (due to mobiles in other cells) is proportional to intra-cell interference (due to mobiles inside the cell). We denote by $\delta$ the ratio of inter-cell interference to intra-cell interference. The results may easily be extended to models where inter-cell interference is taken into account in a more accurate way.

\subsection{Feasibility condition}

We first assume that all mobiles require the same service, characterized by a constant target SNR $\xi$. Let $P_{l}$ be the transmission power of a mobile in state $l$ and $\Gamma_{l}$ its path loss. The SNR received by the base station from a mobile in state $l$ is equal to:

$$
\mathrm{SNR}_{l}=\frac{P_{l} \Gamma_{l}}{N_{0}+(1+\delta)\left(\sum_{j \in \mathcal{L}} n_{j} P_{j} \Gamma_{j}-P_{l} \Gamma_{l}\right)}
$$

where $N_{0}$ denotes the thermal noise power. Let $P$ be the maximum transmission power of each mobile. The maximum SNR that can be achieved by all 
mobiles is obtained by equalizing the received powers $P_{l} \Gamma_{l}$. It is determined by the mobile with the worst radio conditions as follows:

$$
\mathrm{SNR}=\frac{P \Gamma(n)}{N_{0}+(1+\delta)(|n|-1) P \Gamma(n)} \quad \text { with } \quad \Gamma(n)=\min _{l: n_{l}>0} \Gamma_{l} .
$$

No power constraint. If $P$ is infinite, the feasibility condition is

$$
\frac{1}{(1+\delta)(|n|-1)} \geq \xi
$$

so that the maximum number of mobiles in the cell is:

$$
M=1+\frac{1}{(1+\delta) \xi} .
$$

There is no outage and the blocking probability is simply given by the Erlang loss formula [9].

Power constraint. If $P$ is finite, it follows from (17) that the maximum number of mobiles in the cell is:

$$
M(n)=1+\frac{1}{1+\delta}\left(\frac{1}{\xi}-\frac{1}{\xi(n)}\right) \quad \text { with } \quad \xi(n)=\frac{P \Gamma(n)}{N_{0}} .
$$

Note that $\xi(n)$ corresponds to the maximum SNR of the mobile that experiences the worst radio conditions in network state $n$. In particular, $M(n)$ is positive if and only if $\xi(n) \geq \xi$. For a greedy admission policy where calls are admitted as long as the feasibility condition is satisfied, the blocking condition depends on the mobile with the worst radio conditions only. Outage may occur when this mobile gets even worse radio conditions. In the following, we consider the greedy admission policy and the outage policy that minimizes the number of mobiles in outage, which is typically realized by usual power control algorithms. Note that both policies are monotonic.

\subsection{A linear network}

The blocking and outage probabilities depend on the exact network topology. For the sake of clarity, we illustrate the results on regular topologies only. We first consider a linear network where mobiles and base stations are located on a line, with a fixed distance $2 R$ between two adjacent base stations. In a homogeneous radio environment, the state $l$ of a mobile is simply characterized by its distance $r_{l}=R \times l / L$ from the closest base station. The path loss $\Gamma_{l}$

of a mobile in state $l$ is typically inversely proportional to $r_{l}^{\alpha}$, where $\alpha$ is the 
path loss exponent. Denote by $M_{l}$ the maximum number of mobiles when the state of the mobile with the worst radio conditions is $l$. It follows from (19) that

$$
M_{l}=\left\lfloor M_{L}+\left(M_{1}-M_{L}\right) \frac{L^{\alpha}-l^{\alpha}}{L^{\alpha}-1}\right\rfloor .
$$

Note that $M_{1}$ corresponds to the maximum number of mobiles when all mobiles are located at the cell center while $M_{L}$ corresponds to the maximum number of mobiles when at least one mobile is located at the cell edge. We have $M_{L} \leq M_{l} \leq M_{1}$ for all $l \in \mathcal{L}$.

A new call attempted by a mobile in state $l$ is accepted if the number of ongoing calls is less than $M_{l}$ or if there is a mobile in state $k>l$ and the number of ongoing calls is less than $M_{k}$. For a uniform traffic distribution, it follows from (10) that the blocking probability in state $l$ is

$$
B_{l}=1-e^{-\rho}-\sum_{m=1}^{M_{l}-1} e^{-\rho} \frac{\rho^{m}}{m !} \operatorname{Pr}\left[M_{l(m)}>m\right],
$$

where $l(m)$ denotes the maximum of $m$ independent random variables, uniformly distributed over $\{1, \ldots, L\}$. We deduce the following expression for large $L$ :

$$
B_{l}=1-\sum_{m=0}^{M_{L}-1} e^{-\rho} \frac{\rho^{m}}{m !}-\sum_{m=M_{L}}^{M_{l}-1} e^{-\rho} \frac{\rho^{m}}{m !}\left(\frac{M_{1}-m-1}{M_{1}-M_{L}}\right)^{m / \alpha}
$$

The number of mobiles in outage is minimized by interrupting the mobiles with the worst radio conditions. Specifically, a mobile in state $l$ is interrupted if and only if:

$$
\sum_{j=1}^{l} n_{j}>M_{l}
$$

Note that this condition depends on the number of mobiles in states $1, \ldots, l$ only. For large $L$, the outage probability in state $l$ corresponds to the blocking probability in state $l$ for a virtual network restricted to states $1, \ldots, l$, that is

$$
O_{l}=1-\sum_{m=0}^{M_{l}-1} e^{-\frac{\rho l}{L}} \frac{\left(\frac{\rho l}{L}\right)^{m}}{m !}
$$

As expected, the maximum outage probability is experienced at the cell edge, in which case it coincides with the blocking probability (i.e. $O_{L}=B_{L}$ ). 
The analysis is similar for hexagonal networks, provided each cell is approximated by a disk of radius $R$. Again, the state $l$ of a mobile is characterized by its distance $r_{l}=R \times l / L$ from the closest base station. The main difference is that, for a uniform traffic distribution, the mobiles in states $1, \ldots, l$ represent a fraction $(l / L)^{2}$ of the total traffic intensity, instead of $l / L$ for the linear network. For a large number of states $L$, we get the blocking probability of a mobile in state $l$ :

$$
B_{l}=1-\sum_{m=0}^{M_{L}-1} e^{-\rho} \frac{\rho^{m}}{m !}-\sum_{m=M_{L}}^{M_{l}-1} e^{-\rho} \frac{\rho^{m}}{m !}\left(\frac{M_{1}-m-1}{M_{1}-M_{L}}\right)^{2 m / \alpha},
$$

and the outage probability of a mobile in state $l$ :

$$
O_{l}=1-\sum_{m=0}^{M_{l}-1} e^{-\rho\left(\frac{l}{L}\right)^{2}} \frac{\left(\rho\left(\frac{l}{L}\right)^{2}\right)^{m}}{m !}
$$

Figure 1 gives the overall blocking and outage probabilities as a function of the traffic load $\rho$ for voice traffic $(\xi=-19.5 \mathrm{~dB})$ and video traffic $(\xi=-16.5$ $\mathrm{dB})$. For an interference factor $\delta=0.5$, this corresponds to a maximum of $M=60$ voice calls and $M=30$ video calls, respectively. We take $L=1000$ mobile states, $M_{1}=M$ and $M_{L}=M-5$. These values are representative of a transmission power $P=32 \mathrm{~dB}$, a noise power $N_{0}=-100 \mathrm{~dB}$ and a path loss $\Gamma_{l}=-35-35 \log _{10}(l) \mathrm{dB}$, corresponding to a path loss exponent $\alpha=3.5$. For a target outage probability of $10^{-3}$, we observe that the maximum traffic load is approximately equal to 40 erlangs for voice traffic, 15 erlangs for video traffic. Note that the blocking probability is approximately equal to $10^{-2}$ at such load.
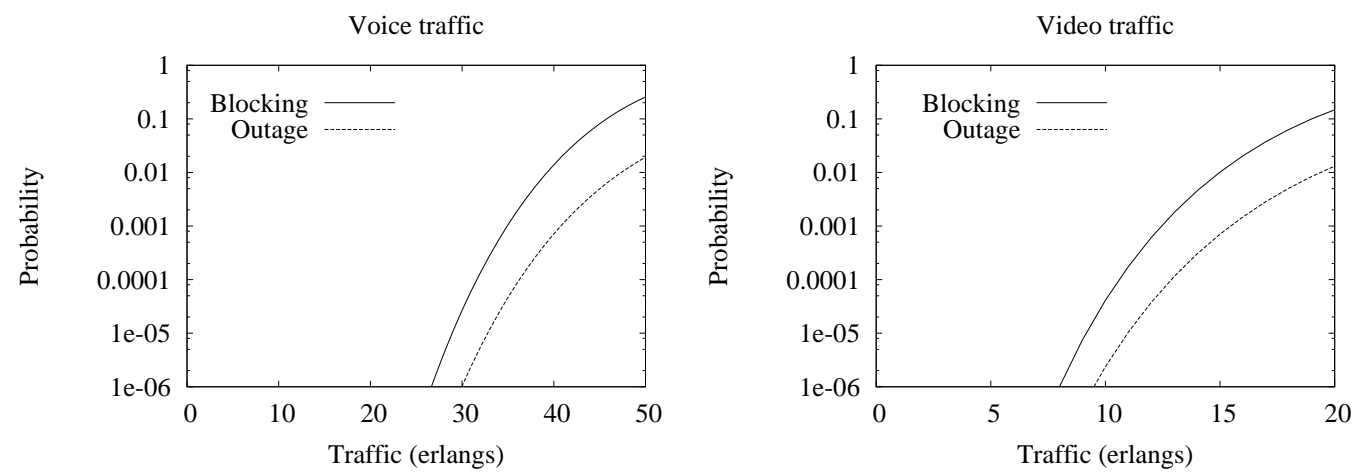

Fig. 1. Blocking and outage w.r.t. traffic intensity (uplink).

While the previous results depend on the marginal distributions of traffic characteristics and radio conditions only, the class structure introduced in Section 2 can be used to evaluate the outage probability of a particular type 
of call. Consider for instance a mobile that moves along a line at constant speed and whose call starts at some cell edge, as shown in Figure 2. Note that mobiles may now go through several cells. Figure 3 gives the corresponding outage probability with respect to the route length (normalized by the cell radius $R$ ). As expected, the outage probability increases significantly when the call ends around some cell edge.

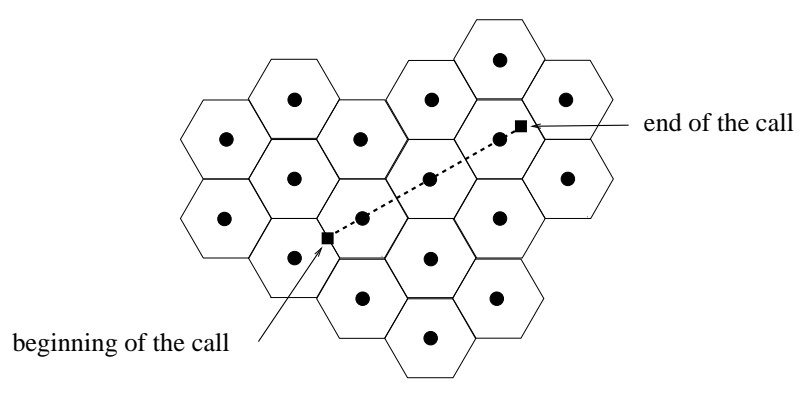

Fig. 2. A particular type of call.
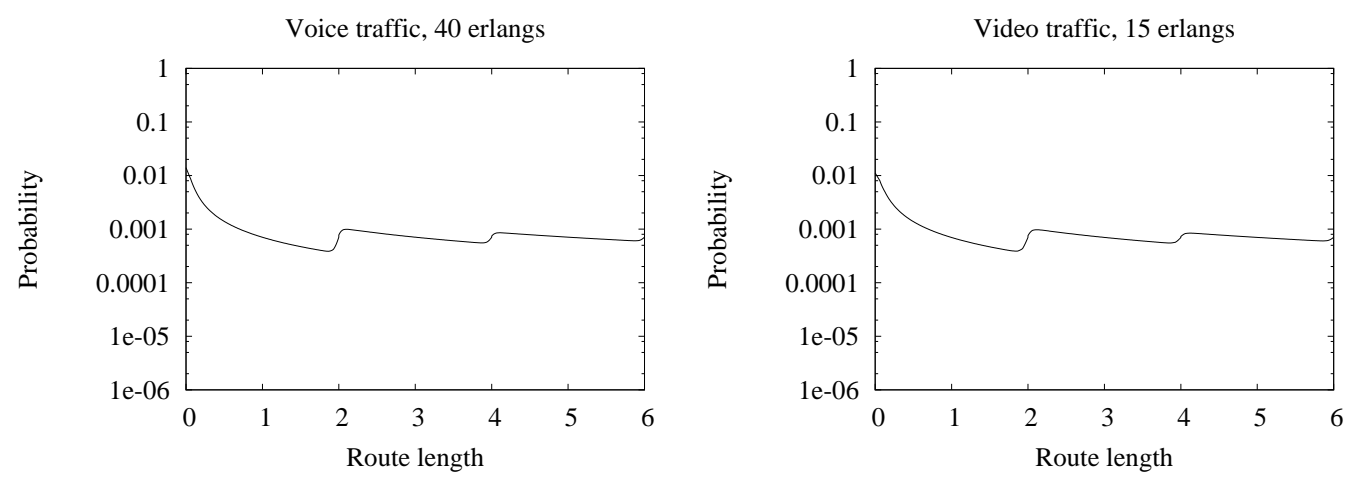

Fig. 3. Outage probability of a particular type of call (uplink).

\subsection{Shadowing}

The radio environment is not homogeneous in practice and the path loss does not only depend on the distance to the closest base station but also on the shadowing due to various obstacles (buildings, trees, cars, etc.) This is usually modelled as a lognormal random variable, so that the actual path loss experienced by a mobile is proportional to $10 \frac{\eta}{10 \sqrt{2}}$ where $\eta$ is a centered normal random variable with standard deviation $\sigma$, typically in the range $4 \mathrm{~dB}$ to 12 $\mathrm{dB}$. The blocking and outage probabilities do not have closed-form expressions in this case but can be numerically evaluated. Figure 4 shows the impact of shadowing for a hexagonal network with the same characteristics as above. We observe that the results are extremely sensitive to the amplitude of the shadowing, which suggests that this parameter should be precisely estimated. 

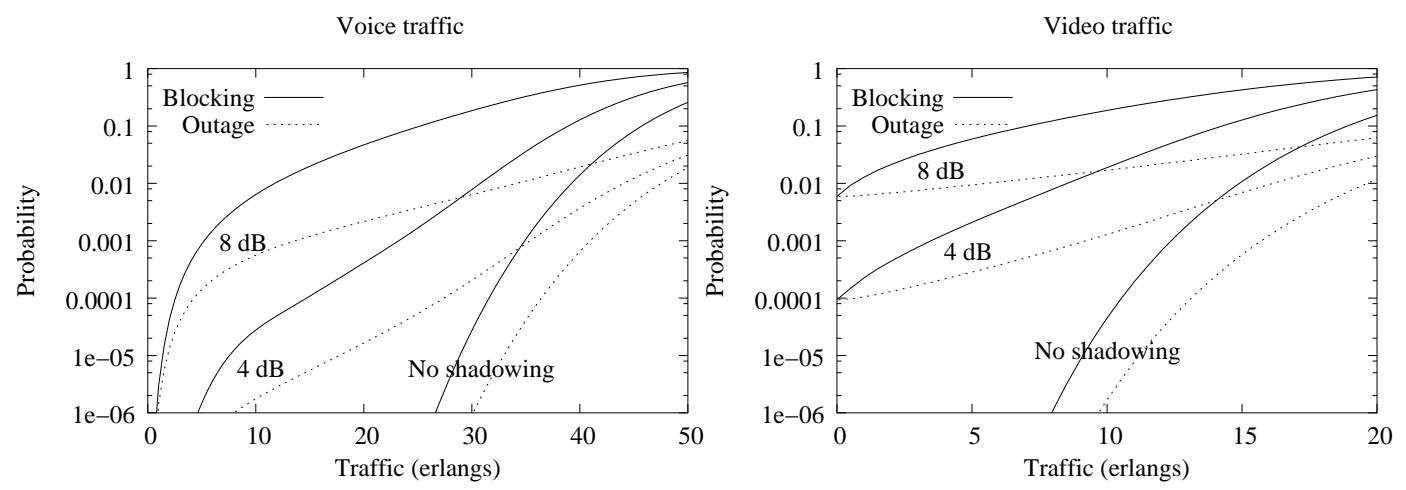

Fig. 4. Impact of shadowing on blocking and outage (uplink, $\sigma=4 \mathrm{~dB}$ and $8 \mathrm{~dB}$ ).

\subsection{Service integration, various power constraints}

The class structure may be used not only to derive the outage probability of a particular type of call (cf. §4.3) but also to evaluate the performance of networks with various types of mobile. Assume class- $k$ mobiles have the same service requirement, characterized by a target SNR $\xi^{k}$, and the same power limit $P^{k}$. The state $l$ of a mobile is still characterized by the path loss $\Gamma_{l}$.

No power constraint. If $P^{k}$ is infinite for all classes $k$, we deduce from (16) the feasibility condition:

$$
\sum_{k \in \mathcal{K}} \frac{n^{k}}{M^{k}} \leq 1 \quad \text { with } \quad M^{k}=1+\frac{1}{(1+\delta) \xi^{k}}
$$

There is no outage and the model reduces to a multirate loss network for which the Kaufman-Roberts recursion applies [17], [19].

Power constraint. If $P^{k}$ is finite for some class $k$, let $\xi^{k}(n)$ be the maximum SNR of the class- $k$ mobile that experiences the worst radio conditions in network state $n$ :

$$
\xi^{k}(n)=\frac{P^{k} \Gamma^{k}(n)}{N_{0}}, \quad \text { with } \quad \Gamma^{k}(n)=\min _{l: n_{l}^{k}>0} \Gamma_{l} .
$$

The feasibility condition becomes:

$$
\sum_{k \in \mathcal{K}} \frac{n^{k}}{M^{k}} \leq \min _{k \in \mathcal{K}} \frac{M^{k}(n)}{M^{k}} \quad \text { with } \quad M^{k}(n)=1+\frac{1}{1+\delta}\left(\frac{1}{\xi^{k}}-\frac{1}{\xi^{k}(n)}\right) .
$$

Figure 5 gives the resulting blocking and outage probabilities for an integrated network with $80 \%$ voice traffic and $20 \%$ video traffic and for a network with voice traffic only but different power constraints (50\% of mobiles with power constraint $P^{1}=29 \mathrm{~dB}, 50 \%$ with power constraint $P^{2}=32 \mathrm{~dB}$ ). The other 
network parameters are the same as in $\S 4.3$. We observe that, unlike the blocking probability, the outage probability is very sensitive to the required service and to the power constraint.
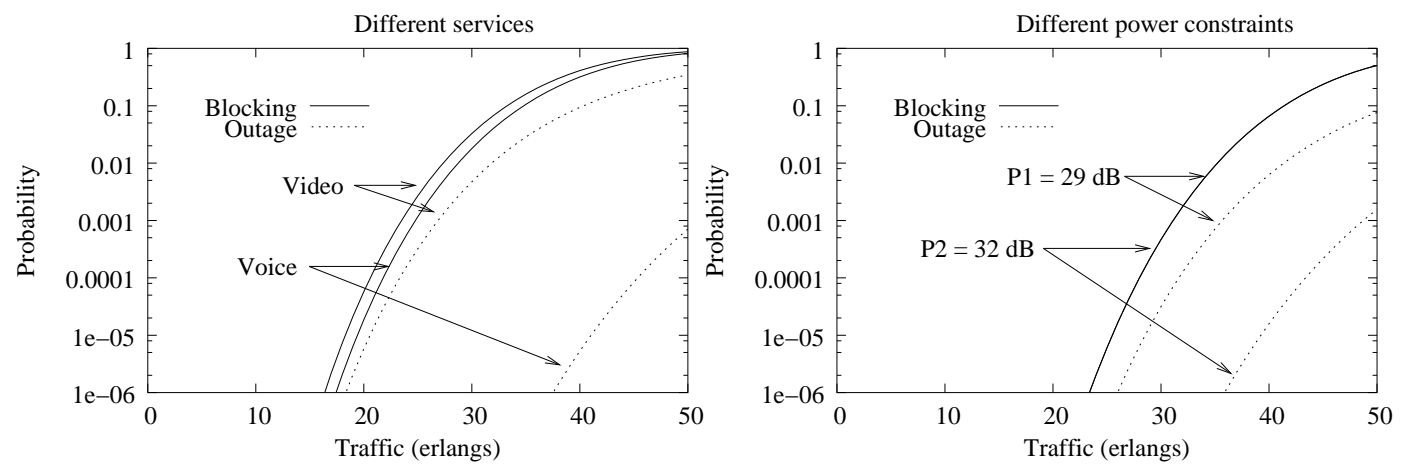

Fig. 5. Impact of service requirement and power constraint on blocking and outage (uplink).

\section{Application to the downlink}

We now apply the results to the downlink of CDMA networks. We assume that all base stations transmit at a constant power, which is representative of cdma2000 EV-DV and UMTS HSDPA systems for instance where the transmission power that is not consumed by real-time traffic on dedicated channels is used by data traffic on a common shared channel (not considered here). Again, the results may well be applied to models where the transmission powers of the base stations vary with time.

\subsection{Feasibility condition}

We focus on a particular cell and denote by $P$ the maximum transmission power of the base station. Let $P_{l} \leq P$ be the transmission power allocated to a mobile in state $l, \Gamma_{l}$ its path loss and $I_{l}$ its inter-cell interference. Denoting by $\gamma$ the orthogonality factor between mobiles in the same cell, the SNR of a mobile in state $l$ is:

$$
\mathrm{SNR}_{l}=\frac{P_{l} \Gamma_{l}}{N_{0}+I_{l}+\gamma\left(P-P_{l}\right) \Gamma_{l}}
$$

Thus for a common target SNR $\xi$, the power required by a mobile in state $l$ is:

$$
P_{l}=\frac{\xi\left(N_{0}+I_{l}+\gamma P\right)}{(1+\gamma \xi) \Gamma_{l}} .
$$


We deduce the feasibility condition:

$$
\sum_{l \in \mathcal{L}} n_{l} P_{l} \leq P
$$

As for the uplink, we evaluate the blocking and outage probabilities for the greedy admission policy and for the outage policy that minimizes the number of mobiles in outage.

\subsection{Blocking and outage}

The feasibility condition (20) defines a multirate loss system for which the Kaufman-Roberts recursion applies [17], [19]. We simply need to assume that the available transmission power $P$ and the transmission power $P_{l}$ required by a mobile in state $l$ are integers, which is not a restrictive assumption as discussed in [17], [19]. For any integer $m$, define:

$$
\omega(m)=\sum_{n: \sum_{l \in \mathcal{L}} n_{l} P_{l}=m} \prod_{l \in \mathcal{L}} \frac{\rho_{l}^{n_{l}}}{n_{l} !} \quad \text { if } \quad m \geq 0
$$

and $\omega(m)=0$ otherwise. The Kaufman-Roberts recursion gives:

$$
\omega(m)=\frac{1}{m} \sum_{l \in \mathcal{L}} \rho_{l} P_{l} \omega\left(m-P_{l}\right) \quad \text { for } \quad m \geq 1,
$$

from which we deduce the blocking probability of a mobile in state $l$ :

$$
B_{l}=1-e^{-\rho} \sum_{m=0}^{P-P_{l}} \omega(m) .
$$

As for the uplink, for large $L$, the outage probability in state $l$ corresponds to the blocking probability in state $l$ for a virtual network restricted to states $1, \ldots, l$, that is

$$
O_{l}=1-e^{-\frac{\rho l}{L}} \sum_{m=0}^{P-P_{l}} \omega_{l}(m)
$$

where $\omega_{l}$ is the analogue of $\omega$ for the restricted network. Figure 6 gives the results for a hexagonal network with the same parameters as in $\S 4.3$, given a transmission power $P=40 \mathrm{~dB}$ and an orthogonality factor $\gamma=0.5$.

Comparing with Figure 1, we observe that the maximum traffic for given target blocking and outage probabilities is much higher than for the uplink. Again, the shadowing has a significant impact on these results, as illustrated in Figure 7 for the same parameters as those of Figure 4. 

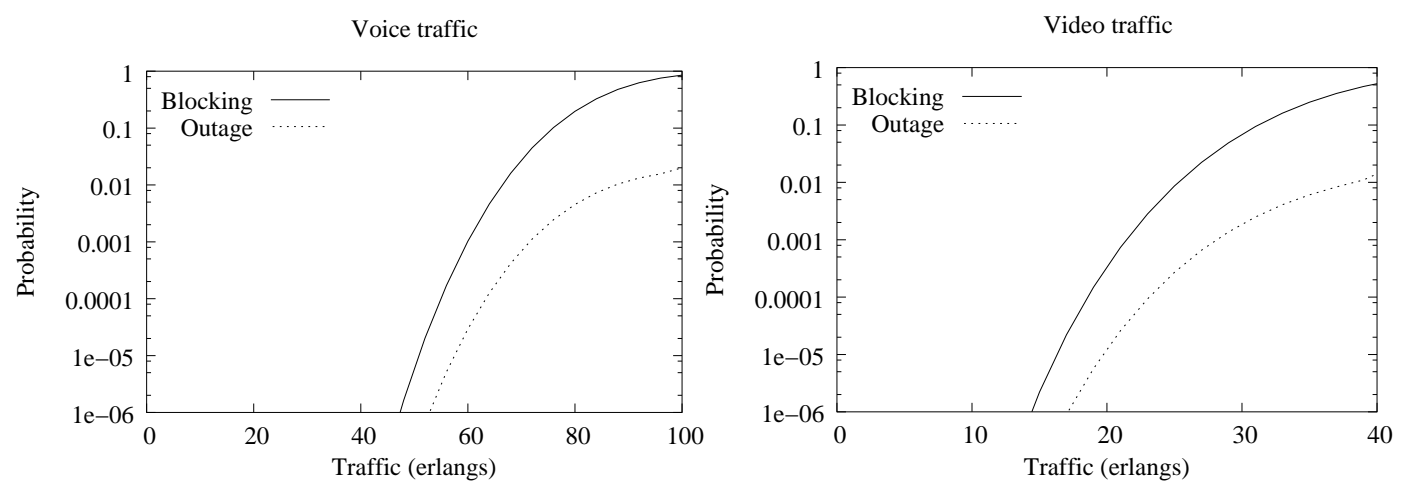

Fig. 6. Blocking and outage w.r.t. traffic intensity (downlink).
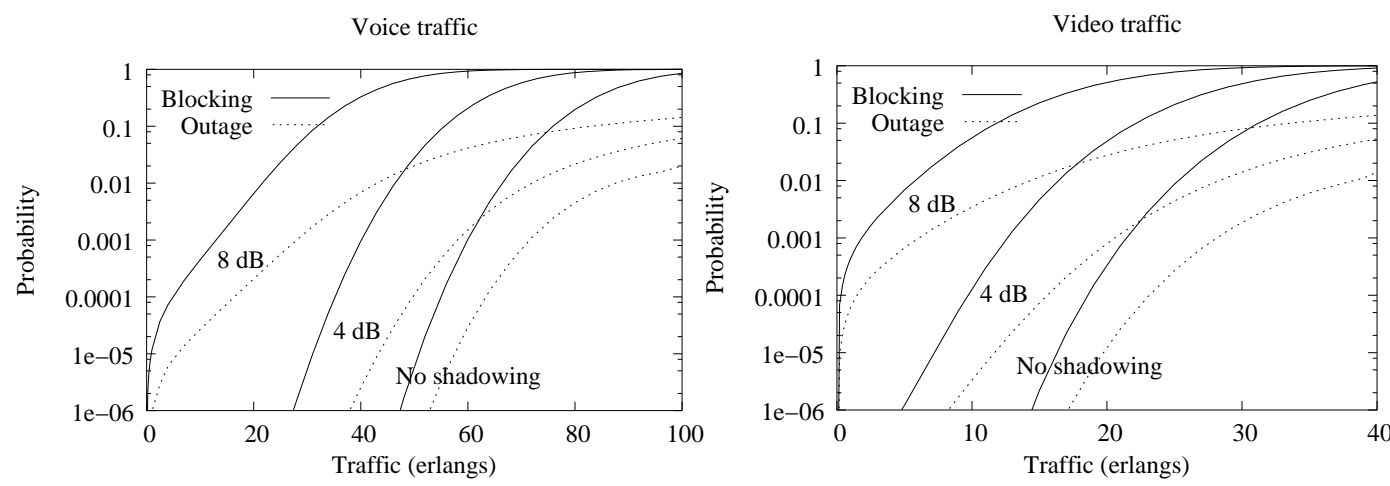

Fig. 7. Impact of shadowing on blocking and outage (downlink, $\sigma=4 \mathrm{~dB}$ and 8 $\mathrm{dB})$.

\subsection{Service integration}

Finally, we use the class structure to evaluate the performance of a multiservice network. Let $\xi^{k}$ be the target SNR of class- $k$ mobiles. The feasibility condition becomes:

$$
\sum_{k \in \mathcal{K}} \sum_{l \in \mathcal{L}} n_{l}^{k} P_{l}^{k} \leq P
$$

where $P_{l}^{k}$ denotes the power required by a class- $k$ mobile in state $l$ :

$$
P_{l}^{k}=\frac{\xi^{k}\left(N_{0}+I_{l}+\gamma P\right)}{\left(1+\gamma \xi^{k}\right) \Gamma_{l}} .
$$

Figure 8 gives the corresponding blocking and outage probabilities for $80 \%$ voice traffic and $20 \%$ video traffic, for the same parameters as above (in the absence of shadowing).

As for the uplink, we observe that the blocking probability is similar for voice calls and video calls whereas the outage probability strongly depends on the service requirement (it is less than $10^{-6}$ for voice calls as long as the traffic intensity is less than 100 erlangs). There is a natural discrimination against 


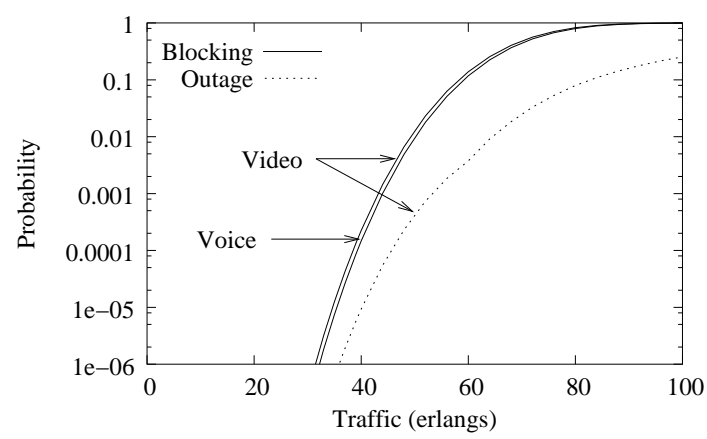

Fig. 8. Impact of service requirement on blocking and outage (downlink).

video calls that consume more radio resources than voice calls and are therefore interrupted first in an outage situation. In particular, there is no need to introduce some form of priority to voice calls over video calls to protect voice calls. It is sufficient to minimize the number of calls in outage, which is typically realized by usual power control algorithms.

\section{Conclusion}

We have presented conservative estimates of the blocking and outage probabilities in a wireless network with varying bit rate requirements and radio conditions (due to mobility for instance). We have considered the outage probability as perceived by users, that is the probability an arbitrary call is in outage, and not the non-feasibility probability, which is the probability at least one call is in outage. Though often referred to as the outage probability as well, the nonfeasibility is rather related to the blocking probability of a virtual call that would not require any radio resource (cf. $\S 3.2$ ). The results of Sections 4 and 5 suggest that the outage probability is typically much less than the blocking probability in practice, but also much more sensitive to call characteristics like the service requirement (e.g. voice or video call).

Though we applied the results CDMA networks only, the method applies to virtually any network configuration, including other access technologies like TDMA and FDMA. The network topology and the traffic characteristics may be quite general. We considered Poisson call arrivals only but it is in fact sufficient to add an idle state in the set $\mathcal{L}$ to represent Poisson session arrivals, each session consisting of an alterning series of calls and periods of inactivity. We may also explore other admission and outage policies. The state of a mobile may well include its past trajectory for instance. The potential gain of using that information to estimate its future trajectory and to design efficient admission and outage policies on this basis could be quantified. The only restriction is that the considered policies must be monotonic. Though the monotonicity property holds in most cases, there are exceptions that are 
worth mentioning. An example is an admission policy where the decision of admitting or blocking a new call is based on the number of admitted calls that are not in outage, and not on the total number of admitted calls. While the method still applies in this case, the considered estimates are not longer guaranteed to be conservative.

Another possible extension of the present work concerns rate-adaptive video applications. The video calls that consume too much radio resources may adapt their bit rate requirement instead of being interrupted. This would reduce the outage probability (and possibly the blocking probability, depending on the admission policy) at the price of a varying video quality. Given the scarceness of the radio resources, the potential net benefit of introducing such adaptive video codecs would be worth quantifying.

\section{References}

[1] J.M. Aein, Power balancing in systems employing frequency reuse, COMSAT Technical Review 3-2 (1973) 277-300.

[2] E. Altman, Capacity of Multi-service Cellular networks with transmission-rate control: A queueing analysis, in: Proc. of ACM Mobicom (2002).

[3] S. Anand, A. Chockalingam, K.N. Sivarajan, Outage and capacity analysis of cellular CDMA with admission control, in: Proc. of IEEE WCNC (2002).

[4] F. Baccelli, B. Blaszczyszyn, M. Karray, Blocking rates in large CDMA networks via a spatial Erlang formula, in: Proc. of IEEE INFOCOM (2005).

[5] F. Berggren, Power control and adaptive resource allocation in DS-CDMA systems Doctoral Thesis, Royal Institute of Technology (2003).

[6] F. Bock, B. Ebstein, Assignment of transmitter powers by linear programming, IEEE Trans. on Electromagnetic Compatibility 6-2 (1964) 36-44.

[7] T. Bonald, A. Proutière, Insensitivity in processor-sharing networks, Performance Evaluation 49 (2002) 193-209.

[8] C.C. Chan, S.V. Hanly, Calculating the outage probability in a CDMA network with spatial Poisson traffic, IEEE Trans. on Vehicular Technology 50-1 (2001) 183-204.

[9] A.K. Erlang, Solution of some problems in the theory of probabilities of significance in automatic telephone exchanges, Elektrotkeknikeren 13 (1917).

[10] J. Evans, D. Everitt, Effective bandwidth-based admission control for multiservice CDMA cellular networks, IEEE Trans. on Vehicular Technology 48-1 (1999) 36-46. 
[11] J.S. Evans, D. Everitt, On the teletraffic capacity of CDMA cellular networks, IEEE Trans. on Vehicular Technology 48-1 (1999) 153-165.

[12] G.J. Foschini, Z. Miljanic, A simple distributed autonomous power control algorithm and its convergence, IEEE Trans. on Vehicular Technology 42-4 (1993) 641-646.

[13] K.S. Gilhousen, I.M. Jacobs, R. Padovani, A.J. Viterbi, L. A. Weaver, C. E. Wheatley, On the capacity of a cellular CDMA system, IEEE Trans. on Vehicular Technology 40 (1991) 303-312.

[14] S.V. Hanly, Information capacity of radio networks, PhD thesis, King's college, Cambridge Univ. (1993).

[15] W.S. Jeon, D.G. Jeong, Call admission control for CDMA mobile communications systems supporting multimedia services, IEEE Trans. on Wireless Communications 1-4 (2002) 649-659.

[16] S. Kandukuri, S. Boyd, Optimal power control in interference-limited fading wireless channels with outage probability specifications, IEEE Trans. on Wireless Communications 1 (2002) 46-55.

[17] J.S. Kaufman, Blocking in a shared resource environment, IEEE Trans. on Communications 29 (1981) 1474-1481.

[18] F.P. Kelly, Reversibility and Stochastic Networks (Wiley, 1979).

[19] J.W. Roberts, A service system with heterogeneous user requirement, in: G. Pujolle, eds., Performance of Data Communications Systems and Their Applications (North-Holland, 1981) 423-431.

[20] A.J. Viterbi, A.M. Viterbi, Erlang capacity of a power controlled CDMA system, IEEE J. Select. Areas Comm. 11 (1993) 892-900.

[21] J. Zander, Performance of optimum transmitter power control in cellular radio systems, IEEE Trans. on Vehicular Technology 41 (1992) 57-62.

Thomas Bonald graduated from Ecole Polytechnique in 1994 and qualified as an engineer at Ecole Nationale Supérieure des Télécommunications in 1996. He has a $\mathrm{PhD}$ in applied mathematics from Ecole Polytechnique, his graduate research being performed at INRIA in the area of network flow control. He is jointly with the traffic modelling group of Jim Roberts at France Telecom and the network theory group of Francois Baccelli at Ecole Normale Supérieure, working on stochastic models of fixed and wireless networks.

Alexandre Proutière graduated from Ecole Normale Supérieure in 1996 and qualified as an engineer at Ecole Nationale Supérieure des Télécommunications in 1998. He has a $\mathrm{PhD}$ in applied mathematics from Ecole Polytechnique, his graduate research being performed at France Telecom in the area of quality of service in integrated networks. He is jointly with the traffic modelling group of Jim Roberts at France Telecom and the network theory group of Francois Baccelli at Ecole Normale Supérieure, working on stochastic models of fixed and wireless networks. 